\title{
Lee Silverman voice treatment (LSVT) mitigates voice difficulties in mild Parkinson's disease
}

\author{
Arezoo Saffarian ${ }^{1}$, Yunes Amiri Shavaki ${ }^{1}$, Gholam Ali Shahidi ${ }^{2}$, Shahram Hadavi ${ }^{1}$, Zahra Jafari*3 \\ Received: 27 July 2018 \\ Published: 13 Feb 2019
}

\begin{abstract}
Background: Parkinson's disease (PD) is a progressive neurological disorder and many PD patients experience some type of voice and speech disorders during the course of illness. In this study, the aim was to investigate the effect of Lee Silverman voice treatment (LSVT) on improving voice difficulties in patients with mild PD using voice handicap index (VHI).

Methods: This interventional study was conducted on 23 PD patients who were randomly divided into 2 groups: a treatment group $(\mathrm{PD}-\mathrm{T})(\mathrm{n}=13)$ and a no-treatment group (PD-NT) $(\mathrm{n}=10)$. Neurologically healthy control (NNC) group consisted of 13 healthy participants who did not suffer from voice and speech problems and were matched with PD group by age (50-65 years), sex, and education. VHI questionnaire was completed a day before the start of LSVT and a day after the treatment fulfillment for the PD-T group; the same time spots were applied for the PD-NT and NNC groups. Statistical analyses were performed using SPSS Statistics 22.0 and significance level was set at 0.05 . The multivariate analysis of variance and repeated measure analysis of variance were used for data analysis.

Results: PD groups showed a significant weakness in VHI scores before treatment compared to NNC group $(\mathrm{p} \leq 0.001)$. The mean of VHI scores for PD-T, PD-NT, and NNC groups before treatment was $44.31 \pm 11.23,43.54 \pm 6.10$, and $8.15 \pm 4.27$, respectively. LSVT was successful in improving VHI scores in PD-T group $(17.23 \pm 5.35, \mathrm{p} \leq 0.001)$. However, no improvement was observed in PD-NT group (44.00 \pm 5.88$)$.
\end{abstract}

Conclusion: Improvement in VHI score could be the result of ameliorated self-monitoring and self-regulation created by LSVT.

Keywords: Lee Silverman voice treatment, Parkinson's disease, Voice disorder

Conflicts of Interest: None declared

Funding: Iran University of Medical Sciences

*This work has been published under CC BY-NC-SA 1.0 license.

Copyright $\odot$ Iran University of Medical Sciences

Cite this article as: Saffarian A, Amiri Shavaki Y, Shahidi GA, Hadavi Sh, Jafari Z. Lee Silverman voice treatment (LSVT) mitigates voice difficulties in mild Parkinson's disease. Med J Islam Repub Iran. 2019 (13 Feb);33:5. https://doi.org/10.47176/mjiri.33.5

\section{Introduction}

Parkinson's disease (PD) is a chronic neurodegenerative disorder characterized by progressive loss of dopaminergic neurons in the substantia nigra. PD is the second most common neurodegenerative disorder after Alzheimer's disease (1). In addition to motor symptoms (tremor, rigid muscles, bradykinesia), many PD patients also suffer from non-motor deficits, such as mood and behavioral changes, language impairment, sleep disorders, cognitive dysfunction, and speech impairment known as hypokinetic dysar-

Corresponding author: Dr Zahra Jafari, jafari.z@iums.ac.ir

1. Department of Speech Therapy, School of Rehabilitation Sciences, Iran University of Medical Sciences, Tehran, Iran

2. Movement Disorders Clinic, Hazrat Rasool Hospital, Iran University of Medical Sciences, Tehran, Iran

3. Department of Basic Sciences in Rehabilitation, School of Rehabilitation Sciences, Iran University of Medical Sciences, Tehran, Iran thria (2). Approximately, $70 \%$ to $90 \%$ of patients with PD show some type of speech disorders during the course of the disease (3-5). These disorders may be one of the primary indicators of PD (6), however, only $37 \%$ of PD patients receive speech therapy (7).

Dysarthria associated with PD can be treated with medication and surgical treatments, such as deep brain stimulation of subthalamic nucleus (STN-DBS) and speech therapy methods (8). Over the years, various methods have

$\uparrow$ What is "already known" in this topic:

The Voice Handicap Index (VHI) has been found to be sensitive to various voice difficulties as shown in Parkinson's disease (PD). VHI has been applied to measure the effectiveness of diverse treatments in improving voice and speech disorders in PD.

\section{$\rightarrow$ What this article adds:}

This study detected improvements in VHI scores, which may be due to the ameliorated self-monitoring and self-regulation developed by LSVT. 
been presented by speech and language pathologists to improve communication in PD patients. Conventional treatments involved speed-controlling strategies to improve the intelligibility of speech (9) and alternative communicative strategies (10). Reviewing articles discussing the effectiveness of these treatments showed varied results, and the continuity and generalizability of the acquired skills were also limited (11). The Lee Silverman voice treatment (LSVT) is one of the most widely used speech intervention methods in hypokinetic dysarthria associated with PD (12), with which PD patients have achieved short- and long-term therapeutic results (13-15). The 5 main concepts in the LSVT are as follow: (1) focusing on loudness, (2) high-effort vocalization, (3) intensive therapy plan, (4) calibration or knowledge of the amount of effort needed to raise the voice, and (5) quantification or evaluation of the patient's performance as a tool for motivation (16). LSVT both improves vocal loudness and reduces the negative effects of $\mathrm{PD}$ on communication. Also, it positively affects speech intelligibility, pitch changeability, speed, and vocal cords adduction (16-19). LSVT is an intensive speech treatment, conducted individually and by a trained speech and language pathologist. LSVT program targets vocal loudness and increases phonation efforts through various speech exercises and selfmonitoring (19). The purpose of LSVT is to enable the patient to automatically use a louder and a good-quality voice in everyday life $(14,16,17,20,21)$.

It is highly important to examine the vocal changes associated with the duration of the disorder or previous treatments (medical, surgical, behavioral) (22). Voice Handicap Index (VHI) is a tool to quantify the patient's perception of his/her own impairment due to vocal problems (23). The effects of voice handicap disabilities on quality of life (QoL) have been shown in different studies $(24,25)$. VHI has been proved to be sensitive to various voice disorders, such as in PD patients (23, 26-32). In a recent study by Guimaraes et al, VHI was used as a valid and reliable tool in patients with mild to moderate PD, however, further research is required for severe PD cases (33). In addition, this questionnaire has been used to study the effectiveness of various treatments in improving voice and speech disorders in PD (34-36), for example, the effect of LSVT on voice disorders (7, 37-41).

Taking into account the growth in the elderly population in most countries across the world, the prevalence of neurodegenerative diseases accompanying communication disorders is also rising (42).

Thus, nowadays, rehabilitation sciences should focus on early identification and management of hearing, speech, and language disorders in chronic aging diseases, such as PD. Previous studies have already shown the usefulness of LSVT program for patients with PD severity of higher than 2 (according to the diagnostic H\&Y scale) (7, 37-41). However, to date, no research has demonstrated to what extent this well-known voice therapy method could be helpful to those in the early stage of the disease. Thus, in this study, it was hypothesized that an early LSVT program could be more advantageous in relieving voice disorders, assessed with VHI, in patients with a mild PD compared to a control matched group.

\section{Methods}

Participants: This interventional study was conducted on a group of PD patients and a healthy neurological control group (NNC) aged 50-65 years. Patients with PD included 23 participants who were randomly assigned into the treatment group (PD-T, $n=13$, mean age $=56.64 \pm 4.70$ years, 7 females and 6 males) and no-treatment group (PD-NT, $\mathrm{n}=10$, mean age $=57.82 \pm 3.46$ years, 5 females and 5 males). The names of the patients were recorded based on a number and they were randomly divided into 2 groups by a person who was unaware of the research plan. The NNC group consisted of 13 healthy participants (mean age $=56.14 \pm 3.30$ year, 7 females and 6 males) who did not suffer from voice and speech problems. They were matched with PD patients by age, sex, education, cognitive status, and level of depression. Inclusion and exclusion criteria for the NNC group were similar to those described for the patient group (Table 1).

A total of 23 patients with idiopathic PD (IPD), diagnosed by a neurologist using UK Parkinson's Disease Society Brain Bank clinical diagnostic criteria, were included in the study (43). Inclusion criteria were as follow: disease severity of stages 1 and 2 (mild) (44, 45) according to the Hoehn \& Yahr scale (46); diagnosis of IPD 1 to 8 years earlier (mean duration since diagnosis= $4.67 \pm 1.24$ years) (47); and using Levodopa medication $(48,49)$. Moreover, the patient's medication status did not change during the assessment period and LSVT $(18,50)$, participants had to be monolingual native Persian speakers, right-handed $(8,51)$, have a normal or modified range hearing (14), and have complaints about voice and speech (7).

Exclusion criteria were as follow: history of stroke, head trauma or brain surgery $(48,52)$; using treatments other than medications, such as L-dopa (including DBS surgery of the STN) $(48,51,52)$; and a history of laryngeal surgery or damage $(7,51)$. Furthermore, individuals with depression severity of above 10 , according to the geriatric depression scale (GDS) (53), and those with impaired general cognitive ability according to Mini-mental State Examination (MMSE) test (score of less than 26 of the maximum score of 30) (5457) were excluded from the study. None of the patients had undergone any other type of speech therapy before or during this study (5).

The control group consisted of 13 healthy participants (7 females and 6 males) who did not have any history of voice and speech problems or speech therapy. They were $50-65$ years old, with a mean age of $56.14 \pm 3.30$ years. According to the speech and language pathologist, they did not suffer from any voice problem and were matched with the PD patients by age, sex, and education.

This study was supported by Iran University of Medical Sciences (grant number: 6114/105/ $/ 93$ ). All patients completed the consent form to participate in this study. Treatment was conducted free of charge for all patients. The Persian version of the VHI questionnaire (58) was completed by the patient a day before the start of treat- 
ment and a day after the study fulfillment. Assistance was provided to patients by one of the research team members if they needed help to complete the questionnaire.

\section{Voice Handicap Index}

The VHI questionnaire is self-administered and consists of 30 questions, which are scored from 0 (never) to 4 (always). These 30 questions are equally divided into 3 aspects: functional, physical, and emotional aspects of voice disorders. The functional subscale (VHIF) examines the impact of voice problems on the everyday life of the individual. The physical subscale (VHIP) evaluates the patient's self-perception of laryngeal discomfort and voice output characteristics. The emotional subscale (VHIE) assesses the patient's emotional responses (30).

VHI is scored between 0 to 120 , with 120 indicating the maximum inability perceived by the patient due to voice problems. Each subscale is scored between 0 to a maximum of 40 (36). The Persian version of the VHI questionnaire was used in this research (58). The results of the study revealed a high internal consistency (high Cronbach's $\alpha$ for the total score (0.86), physical (0.84), emotinal (0.91), and functional (0.86) subscales). Also, a significant discrimination coefficient and high clinical validity and reliability of the Persian VHI was confirmed in this study (58).

\section{Voice treatment}

LSVT was conducted on PD patients by one of the authors (A.S.), certified by LSVT Global Inc., according to its main treatment protocol, published by Ramig et al (16). This treatment consists of 16 individual therapy sessions (four 1-hour sessions per week for 4 successive weeks), which was performed very similarly for all patients. This treatment comprises of multiple repetitions (15 times and more), maximum effort of the individuals, and daily tasks. The complexity of the exercises increases during 4 weeks by increasing the cognitive/motor load of the exercises and increasing the duration and difficulty of the speech assignments. Exercises can be done at home (2). The treatment program was performed individually in a quiet room. Clinical assignments used in LSVT were first translated into Persian by a speech and language pathologist who was fluent in both English and Persian (39).

\section{Statistical analysis}

All statistical analyses were performed using SPSS Statistics 22.0 at a significance level of 0.05 . Normally distributed data were analyzed using the KolmogorovSmirnov test. Because of the normal distribution of the data ( $\mathrm{p} \geq 0.401)$, parametric statistical tests were applied.
The multivariate analysis of variance was conducted to compare VHI score and its subscales in the 3 groups (PD$\mathrm{T}$, PD-NT and NNC). A repeated measures analysis of variance was conducted to compare VHI score and its subscales in the first and the second assessments in every group. F values, p-values, estimations of the effect size (ES) (partial $\eta^{2}$ ), and observed power were reported for the statistical analyses. An ES of less than 0.05 was considered small, an ES of 0.50-0.79 medium, and an ES of 0.80 or above large (59). The Tukey post-hoc test was performed for multiple comparisons of group means in each measurement.

\section{Results}

The demographic characteristics among the groups studied in this research are presented in Table 1 . The mean MMSE score in the experimental group was 28.35 and 29.57 in the control group. These statistics indicate a lack of significant intergroup difference $(p=0.071)$. The mean severity of disease in the experimental group was 1.46 (mild severity), and the mean depression score was 2.71 in this group. However, this was 1.57 in the control group, suggesting the lack of significant intergroup differences $(\mathrm{p}=0.065)$.

The results of the VHI and its subscales (VHIE, VHIP, VHIF) in the first assessment (before starting the treatment) were compared among the 3 groups. Compared with the NNC group, the PD groups showed significantly higher scores in all measurements, including the VHI $\left(\mathrm{p} \leq 0.000, \eta^{2}=0.836\right.$, power $\left.=1.000\right)$, and its subscales VHIE $\quad\left(p \leq 0.001, \quad \eta^{2}=0.572, \quad\right.$ power $\left.=1.000\right), \quad$ VHIP $\left(\mathrm{p} \leq 0.001, \eta^{2}=0.805\right.$, power $\left.=1.000\right)$, and VHIF $(\mathrm{p} \leq 0.000$, $\eta^{2}=0.847$, power $=1.000$ ) (Fig. 1). No significant difference was observed between the $2 \mathrm{PD}$ groups in the first assessment (VHI, $\mathrm{p}=0.966$; VHIE, $\mathrm{p}=0.815$; VHIP, $\mathrm{p}=0.577$; and VHIF, $\mathrm{p}=0.489$ ) (Fig. 1).

A significant difference was observed between before and after treatment (first and second assessment) results in VHI and its subscales (VHIE, VHIP, VHIF) scores in the PD-T group $(p \leq 0.001)$. However, no significant difference was found between PD-NT $(p=0.054)$ and NNC $(p=0.068)$ groups in the first and the second assessments (Fig. 1).

After LSVT (second assessment), significant differences were observed in VHI scores among the 3 groups $\left(\mathrm{p} \leq 0.001, \eta^{2}=0.903\right.$, power $\left.=1.000\right)$ and its subscales: VHIE $\quad\left(p \leq 0.001, \quad \eta^{2}=0.784, \quad\right.$ power $\left.=1.000\right), \quad$ VHIP $\left(\mathrm{p} \leq 0.001, \eta^{2}=0.849\right.$, power $\left.=1.000\right)$, and VHIF $(76$, $\mathrm{p} \leq 0.001, \eta^{2}=0.853$, power $\left.=1.000\right)$ (Fig. 1).

\section{Discussion}

In this study, the effect of LSVT on improving voice difficulties was examined using the VHI in patients with

Table 1. The demographic characteristics of the 3 groups

\begin{tabular}{lcccccccccc}
\hline \multirow{2}{*}{ Groups } & \multicolumn{2}{c}{ Age (year) } & \multicolumn{2}{c}{ Education (year) } & \multicolumn{2}{c}{ GDS score } & \multicolumn{2}{c}{ MMSE score } & \multicolumn{2}{c}{ Disease severity (H\&Y) } \\
\cline { 2 - 9 } & Mean & SD & Mean & SD & Mean & SD & Mean & SD & Mean & SD \\
\hline PD-T & 56.64 & 4.70 & 15.32 & 2.57 & 2.71 & 0.91 & 28.37 & 0.91 & 0.49 \\
PD-NT & 57.82 & 3.46 & 15.87 & 1.97 & 2.60 & 0.84 & 28.10 & 0.87 & 1.20 \\
NNC & 56.14 & 3.30 & 15.64 & 1.82 & 1.57 & 0.71 & 29.57 & 0.64 & - & -34 \\
\hline
\end{tabular}

GDS, geriatric depression scale; MMSE, mini-mental state examination; NNC, neurologically normal controls; PD-T, Parkinson's disease-treatment; PD-NT, Parkinson's disease no-treatment. 

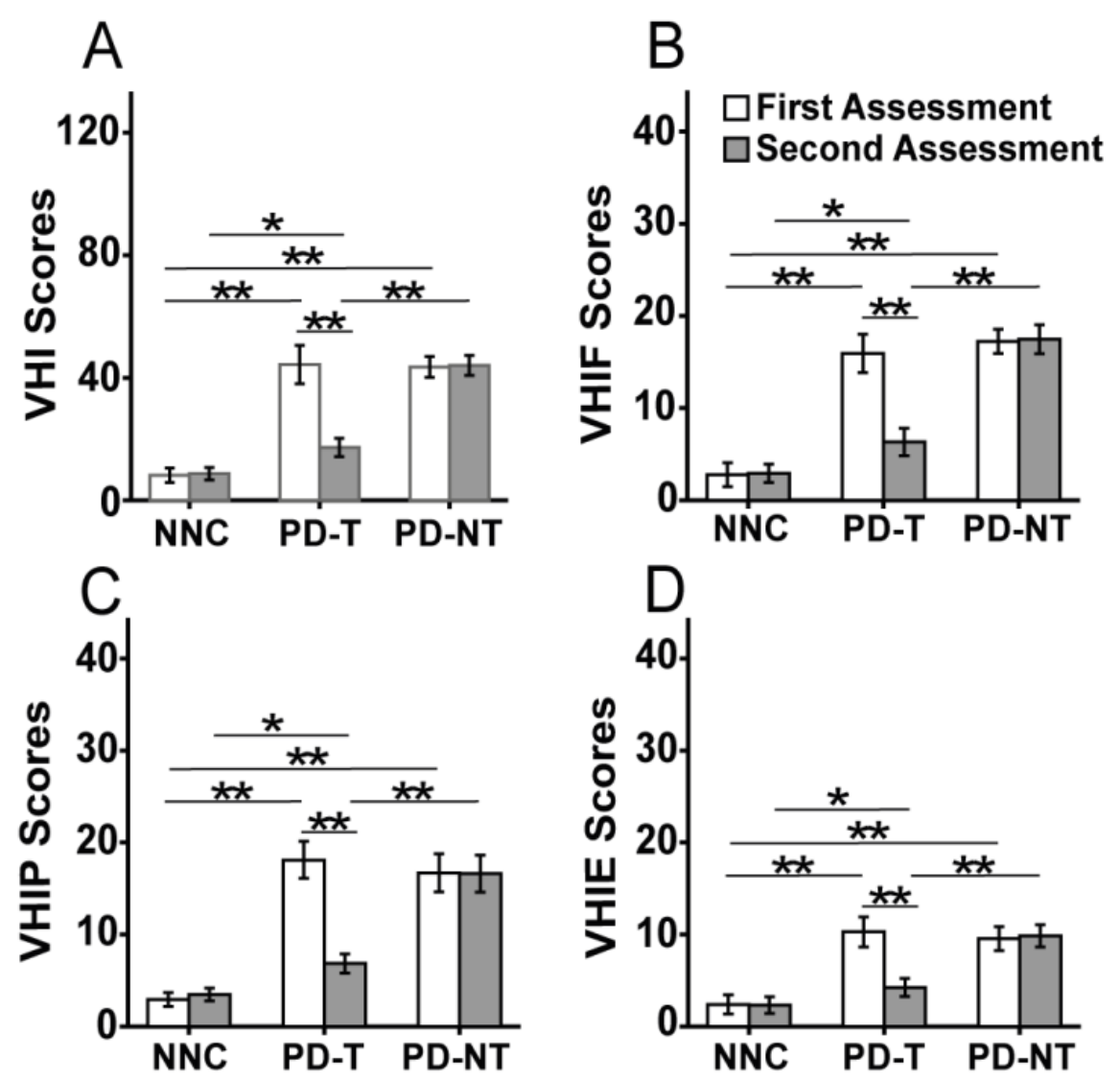

Fig. 1. Results of VHI and its subscales in the first (before LSVT for the PD-T group) and the second (after LSVT for the PD-T group) assessments were demonstrated in the 3 groups. (A) VHI, (B) VHIF, (C) VHIP, and (D) VHIE.

LSVT, Lee Silverman voice treatment; NNC, neurologically normal controls; PD-T, Parkinson's disease-treatment; PD-NT, Parkinson's disease no-treatment; VHI, voice handicap index; VHIF, VHI functional; VHIP, VHI physical; VHIE, VHI emotional. Error bars represent standard error $( \pm \mathrm{SE})$ around the means. Asterisk indicates ${ }^{* *} \mathrm{p}<0.001$ or ${ }^{*} \mathrm{p}<0.05$.

mild PD. The main findings of this study were as follow: (1) The scores on the VHI and its subscales in patients with mild PD were significantly higher compared to the control group; (2) the scores on the VHI and its subscales in the PD group demonstrated remarkable improvement after the LSVT.

Based on findings of previous studies, $78 \%$ of the patients with early PD show some type of vocal disorder $(44,58)$. Assessing patients using VHI, as a tool to study vocal disorders from the individual's perception, showed a significant weakness in PD patients compared to the control group, indicating the presence of vocal disorder in PD patients in the early stages of the disease. In this study, LSVT significantly improved VHI scores in PD patients. This finding indictes that PD patient's perception of his/her voice problems improves after treatment. VHI is a perceptual self-evaluation of the voice that can show the effect of voice problems and related therapies on the patient's quality of life (60), thus, this finding signifies the advantages of LSVT on mitigating voice difficulties in mild PD. LSVT includes 3 important features: (1) voice is the target of the treatment; (2) the mode of the procedure is intensive and high effort is accompanied by neural plasticity principles (19), motor learning theory (61), and skill learning theory (62); and (3) calibration (2). VHIF scores in PD patients in this study improved after treatment. This change could have been due to calibration, which affects the sensory, internal cueing, and neuropsychological deficits and leads to generalization of the treatment outside the therapy session and to other speech situations (2). In fact, LSVT seems to help the patient to recognize the effort needed for a loud voice and to use it in everyday life (2), which results in improved VHIF scores in this study.

As presented in previous studies, LSVT is effective in improving laryngeal function (18). Improvement of laryngeal function in PD patients participated in this study may have played a role in improving VHIP scores after LSVT treatment. Furthermore, LSVT can modify voice quality, vocal loudness, speech quality, and speech intellgibility (2). Therefore, the patient's self-perception of his/her own voice and vocal disorder (VHIE) may improve after LSVT. This finding is in line with previous studies that examined the effect of LSVT on VHI in patients with more severe PD (H\&Y $\geq 2)(37-41)$.

In this study, LSVT significantly improved the scores of VHI and its subscales in PD-T group, but the score of this group still exhibited a noticeable difference with those of the NNC group. This difference might have been due to the abnormal neural drive to speech periphery and abnormal sensorimotor gating (2), which may not be entirely improved even by early LSVT intervention. This hypothesis, however, needs to be investigated in future neuroimaging and neurobehavioral studies. Recent studies 
support the effectiveness of rehabilitation programs, such as LSVT, in early stages of the PD $(63,64)$, as early treatment may slow down the progression of speech syptoms; and if done before other diseases (depression, fatigue, dimentia) worsen, it can lead to optimal treatment outcomes (2).

\section{Limitations}

The number of participants in this study was low, as patients mostly referred for treatment in advanced stages of the disease, and it was difficult to find patients with mild PD who also had other inclusion criteria. Moreover, monitoring the long-term effects of the treatment can provide useful information about the durability of LSVT program in the long-term $(13,14)$, which was not possible due to patients' lack of cooperation. Thus, investigating the longterm effects of LSVT is suggested in the early stages of PD.

\section{Conclusion}

This study showed improvement of VHI and its subscales by LSVT in patients with mild PD, which may be the result of improvements in perception of one's own voice through self-monitoring and self-regulation created by LSVT.

\section{Acknowledgments}

This study was part of a Ph.D. dissertation in SpeechLanguage Pathology (SLP) and was approved by Iran University of Medical Sciences (Grant\# 93/D/320/4189). Cooperation of all participants in this study is greatly appreciated.

\section{Conflict of interests}

The authors declare that they have no competing interests.

\section{References}

1. Launer L, Berger $\mathrm{K}$, Breteler M, Dartigues J, Baldereschi M, Fratiglioni L, et al. Prevalence of Parkinson's disease in Europe: A collaborative study of population-based cohorts. Neurologic Diseases in the Elderly Research Group. Neurology. 2000;54(11 Suppl 5):S21-3.

2. Sapir S, Ramig LO, Fox CM. Intensive voice treatment in Parkinson's disease: Lee Silverman voice treatment. Expert Rev Neurother. 2011;11(6):815-30.

3. Pahwa R, Lyons KE, Kuller WC. Handbook of Parkinson's Disease . $4^{\text {th }}$. New York: NY:Informa Healthcare; 2007.

4. Ho AK, Iansek R, Marigliani C, Bradshaw JL, Gates S. Speech impairment in a large sample of patients with Parkinson's disease. Behav Neurol. 1999;11(3):131-7.

5. Saffarian A, Shavaki YA, Shahidi GA, Jafari Z. Effect of Parkinson Disease on Emotion Perception Using the Persian Affective Voices Test. J Voice. 2018 May 4:S0892-1997(17)30211-4.

6. Duffy J. Motor Speech Disorders: Substrates, Differential Diagnosis and Management Elsevier Health Sciences. 2013.

7. Sackley CM, Smith CH, Rick C, Brady MC, Ives N, Patel R, et al. Lee Silverman voice treatment versus standard NHS speech and language therapy versus control in Parkinson's disease (PD COMM pilot): study protocol for a randomized controlled trial. Trials. 2014;15(1):213.

8. Narayana S, Fox PT, Zhang W, Franklin C, Robin DA, Vogel D, et al. Neural correlates of efficacy of voice therapy in Parkinson's disease identified by performance-correlation analysis. Hum Brain Mapp. 2010;31(2):222-36.
9. Dagenais PA, Southwood MH, Lee TL. Rate reduction methods for improving speech intelligibility of dysarthric speakers with Parkinson's disease. J Med Speech Lang Pathol. 1998;6(3):143-57.

10. Hammen VL, Yorkston KM. Speech and pause characteristics following speech rate reduction in hypokinetic dysarthria. Clin Commun Disord. 1996;29(6):429-45.

11. Yorkston KM. Treatment efficacy: dysarthria. J Speech Lang Hear Res. 1996;39(5):S46-S57.

12. Herd CP, Tomlinson CL, Deane KH, Clarke CE. Comparison of speech and language therapy techniques for speech problems in Parkinson's disease. Cochrane Database Syst Rev. 2012;8.

13. Ramig L, Sapir S, Countryman S, Pawlas A, O'brien C, Hoehn M, et al .Intensive voice treatment (LSVT $\left.{ }^{\circledR}\right)$ for patients with Parkinson's disease: a 2 year follow up. J Neurol Neurosurg Psychiat. 2001;71(4):493-8.

14. Ramig LO, Sapir S, Fox C, Countryman S. Changes in vocal loudness following intensive voice treatment (LSVT $®$ ) in individuals with Parkinson's disease: A comparison with untreated patients and normal age-matched controls. Mov Disord. 2001;16(1):79-83.

15. Sapir S, Ramig LO, Hoyt P, Countryman S, O’Brien C, Hoehn M. Speech loudness and quality 12 months after Intensive Voice Treatment (LSVT®) for Parkinson's disease: a comparison with an alternative speech treatment. Folia Phoniatr Logop. 2002;54(6):296303.

16. Ramig LO, Countryman S, Thompson LL. Horii Y. Comparison of two forms of intensive speech treatment for Parkinson's disease. J Speech Lang Hear Res. 1995a;38:1232-51.

17. Dromey C, Ramig LO, Johnson AB. Phonatory and articulatory changes associated with increased vocal intensity in Parkinson disease: A case study. J Speech Lang Hear Res. 1995;38:751-64.

18. Smith ME, Ramig LO, Dromey C, Perez KS, Samandari R. Intensive voice treatment in Parkinson disease: laryngostroboscopic findings. J Voice. 1995;9(4):453-9.

19. Fox CM, Ramig LO, Ciucci MR, Sapir S, McFarland DH, Farley BG, editors. The science and practice of LSVT/LOUD: neural plasticity-principled approach to treating individuals with Parkinson disease and other neurological disorders. Semin Speech Lang. 2006;27:283-99.

20. Fox CM, Morrison CE, Ramig LO, Sapir S. Current perspectives on the Lee Silverman Voice Treatment (LSVT) for individuals with idiopathic Parkinson disease. Am J Speech-Lang Pathol. 2002;11(2):111-23.

21. Ramig LO, Countryman S, O'Brien C, Hoehn M, Thompson L. Intensive speech treatment for patients with Parkinson's disease Short-and long-term comparison of two techniques.Neurology. 1996;47(6):1496-504

22. Bowling A. Measuring disease: a review of disease-specific quality of life measurement scales: 2nd ed. Buckingham: Open University Press; 1997.

23. Jacobson BH, Johnson A, Grywalski C, Silbergleit A, Jacobson G, Benninger MS, et al. The voice handicap index (VHI): development and validation. Am J Speech-Lang Pathol. 1997;6(3):66-70.

24. Schindler A, Ottaviani F, Mozzanica F, Bachmann C, Favero E, Schettino I, et al. Cross-cultural adaptation and validation of the Voice Handicap Index into Italian. J Voice. 2010;24(6):708-14.

25. Behlau M, dos Santos LdMA, Oliveira G. Cross-cultural adaptation and validation of the voice handicap index into Brazilian Portuguese. J Voice. 2011;25(3):354-9.

26. Hammer MJ, Barlow SM. Laryngeal somatosensory deficits in Parkinson's disease: implications for speech respiratory and phonatory control. Exp Brain Res. 2010;201(3):401-9.

27. Letanneux A, Walshe M, Viallet F, Pinto S. The Dysarthria Impact Profile: a preliminary French experience with Parkinson's disease. Parkinson's Disease. 2013;2013.

28. Madjdinasab F, Cheraghi S, Moradi N, Karkheiran S, Kamali M. Voice Handicap Index (VHI) in Persian Speaking Parkinson's Disease Patients. Iran J Ageing. 2014;9(1):40-45.

29. van Hooren MR, Baijens LW, Vos R, Pilz W, Kuijpers LM, Kremer $\mathrm{B}$, et al. Voice-and swallow-related quality of life in idiopathic Parkinson's disease. Laryngoscope. 2016;126(2):408-14.

30. Louis ED, Gerbin M. Voice handicap in essential tremor: a comparison with normal controls and Parkinson's disease. Tremor Other Hyperkinetic Mov. 2013;3.

31. Bauer V, Alerić Z, Jančić E, Miholović V. Voice quality in 
Parkinson's disease in the Croatian language speakers. Coll Antropol. 2011;35(2):209-12.

32. Shah P, Gore G. Perception of voice difficulties by individuals with Parkinson disease vis-à-vis their family members using Voice Handicap Index. J Indian Speech Language Hear Assoc. 2016;30(1):12

33. Guimaraes I, Cardoso R, Pinto S, Ferreira J. The psychometric properties of the voice handicap index in people with Parkinson's disease. J Voice. 2017;31(2):258. e13-. e18.

34. Searl J, Wilson K, Haring K, Dietsch A, Lyons K, Pahwa R. Feasibility of group voice therapy for individuals with Parkinson's disease. Clin Commun Disord. 2011;44(6):719-32.

35. Shih LC, Piel J, Warren A, Kraics L, Silver A, Vanderhorst V, et al. Singing in groups for Parkinson's disease (SING-PD): A pilot study of group singing therapy for PD-related voice/speech disorders. Parkinsonism Relat Disor. 2012;18(5):548-52.

36. Rosen CA, Murry T, Zinn A, Zullo T, Sonbolian M. Voice handicap index change following treatment of voice disorders. J Voice. 2000;14(4):619-23.

37. Spielman J, Ramig LO, Mahler L, Halpern A, Gavin WJ. Effects of an extended version of the Lee Silverman Voice Treatment on voice and speech in Parkinson's disease Am J Speech-Lang Pathol. 2007;16(2):95-107.

38. Halpern AE, Ramig LO, Matos CE, Petska-Cable JA, Spielman JL, Pogoda JM, et al. Innovative technology for the assisted delivery of intensive voice treatment (LSVT ${ }^{\circledR}$ LOUD) for Parkinson disease. Am J Speech-Lang Pathol. 2012;21(4):354-67.

39. Martel Sauvageau V, Roy JP, Langlois M, Macoir J. Impact of the LSVT on vowel articulation and coarticulation in Parkinson's disease. Clin Linguist Phon. 2015;29(6):424-40.

40. Wight S, Miller N, Lee S. Voice Treatment for people with Parkinson's: audit of outcomes in a routine clinic. Int $\mathrm{J}$ Lang Commun Disord. 2015;50(2):215-25.

41. El Sharkawi A, Ramig L, Logemann J, Pauloski BR, Rademaker A, Smith C, et al. Swallowing and voice effects of Lee Silverman Voice Treatment (LSVT®): a pilot study. J Neurol Neurosurg Psychiat. 2002;72(1):31-6.

42. Ghadirzadeh M, Fadaievatan R, Akbarikamrani A, Davatgaran K, Hasheminazari S, Mirtorabi D. Road Accident Mortality of the Iranian Elderly from 2006 to 2008. Iran J Aging. 2012;7:23.

43. Hughes AJ, Daniel SE, Kilford L, Lees AJ. Accuracy of clinical diagnosis of idiopathic Parkinson's disease: a clinico-pathological study of 100 cases. J Neurol Neurosurg Psychiatr. 1992;55(3):181-4.

44. Lewis SJ, Dove A, Robbins TW, Barker RA, Owen AM. Cognitive impairments in early Parkinson's disease are accompanied by reductions in activity in frontostriatal neural circuitry. $J$ Neurosci. 2003;23(15):6351-6.

45. Rusz J, Cmejla R, Ruzickova H, Ruzicka E. Quantitative acoustic measurements for characterization of speech and voice disorders in early untreated Parkinson's disease. J Acoust Soc Am. 2011;129(1):350-67.

46. Hoehn MM, Yahr MD. Parkinsonism onset, progression, and mortality. Neurology. 1967;17(5):427.

47. Midi I, Dogan M, Koseoglu M, Can G, Sehitoglu M, Gunal D. Voice abnormalities and their relation with motor dysfunction in Parkinson's disease. Acta Neurol Scand. 2008;117(1):26-34.

48. Sapir S, Spielman JL, Ramig LO, Story BH, Fox C. Effects of intensive voice treatment (the Lee Silverman Voice Treatment [LSVT]) on vowel articulation in dysarthric individuals with idiopathic Parkinson disease: acoustic and perceptual findings. J Speech, Lang Hear Res. 2007;50(4):899-912.

49. Cannito MP, Suiter DM, Chorna L, Beverly D, Wolf T, Watkins J. Speech intelligibility in a speaker with idiopathic Parkinson's disease before and after treatment. J Med Speech Lang Path. 2008;16(4):207-13.

50. Angelis Ed, Mourao L, Ferraz H, Behlau M, Pontes P, Andrade L. Effect of voice rehabilitation on oral communication of Parkinson's disease patients. Acta Neurol Scand. 1997;96(4):199-205.

51. Spielman J, Mahler L, Halpern A, Gilley P, Klepitskaya O, Ramig L. Intensive voice treatment (LSVT ${ }^{\circledR}$ LOUD) for Parkinson's disease following deep brain stimulation of the subthalamic nucleus. Clin Commun Disord. 2011;44(6):688-700.

52. Whitehill TL, Wong LLN. Effect of intensive voice treatment on tone-language speakers with Parkinson's disease. Clin Linguist Phon. 2007;21(11-12):919-25.
53. Malakouti K, Fathollahi P, Mirabzadeh A, Salavati M, Kahani S. Validation of geriatric depression scale (GDS-15) in Iran. Res Med. 2006;30(4):361-9.

54. Ventura MI, Baynes K, Sigvardt KA, Unruh AM, Acklin SS, Kirsch $\mathrm{HE}$, et al. Hemispheric asymmetries and prosodic emotion recognition deficits in Parkinson's disease. Neuropsychologia. 2012;50(8):1936-45.

55. Jafari Z, Esmaili M, Delbari A, Mehrpour M, Mohajerani MH. Poststroke acquired amusia: A comparison between right-and left-brain hemispheric damages. NeuroRehabilitation. 2017(Preprint):1-10.

56. Jafari Z, Esmaili M, Toufan R, Aghamollaei M. Bilingual proficiency and cognitive reserve in Persian-English bilingual older adults. Aging Clin Exp Res. 2015;27(3):351-7.

57. Jafari Z, Omidvar S, Jafarloo F. Effects of ageing on speed and temporal resolution of speech stimuli in older adults. Med J Islam Repub Iran. 2013;27(4):195.

58. Moradi N, Pourshahbaz A, Soltani M, Javadipour S, Hashemi H, Soltaninejad N. Cross-cultural equivalence and evaluation of psychometric properties of voice handicap index into Persian. J Voice. 2013;27(2):258.

59. Becker LA. Effect size (ES). Retrieved September. 2000;9:2007.

60 . Maertens K. The voice handicap index as a tool for assessment of the biopsychosocial impact of voice problems. B-ent. 2007;3(2):616.

61. Fox C, Ramig L, Ciucci M, Sapir S, McFarland D, Farley B. Neural plasticity-principled approach to treating individuals with Parkinson disease and other neurological disorders. Semin Speech Lang. 2006;27:283-99.

61. Ofen-Noy N, Dudai Y, Karni A. Skill learning in mirror reading how repetition determines acquisition. $\mathrm{Cog}$ Brain Res 2003; 17(2):507-21.

62. Tillerson JL, Miller GW. Book Review: Forced Limb-Use and Recovery following Brain Injury. Neuroscientist. 2002;8(6):574-85.

63. Hirsch M, Farley B. Exercise and neuroplasticity in persons living with Parkinson's disease. Eur J Phys Rehabil Med. 2009;45(2):21529.

64. Zigmond MJ, Cameron JL, Leak RK, Mirnics K, Russell VA, Smeyne RJ, et al. Triggering endogenous neuroprotective processes through exercise in models of dopamine deficiency. Parkinsonism Relat Dis. 2009;15:S42-S5. 